\title{
Strigolactones fine-tune the root system
}

\author{
Amanda Rasmussen $\cdot$ Stephen Depuydt $\cdot$ \\ Sofie Goormachtig • Danny Geelen
}

Received: 19 April 2013 / Accepted: 5 June 2013 / Published online: 26 June 2013

(C) Springer-Verlag Berlin Heidelberg 2013

\begin{abstract}
Strigolactones were originally discovered to be involved in parasitic weed germination, in mycorrhizal association and in the control of shoot architecture. Despite their clear role in rhizosphere signaling, comparatively less attention has been given to the belowground function of strigolactones on plant development. However, research has revealed that strigolactones play a key role in the regulation of the root system including adventitious roots, primary root length, lateral roots, root hairs and nodulation. Here, we review the recent progress regarding strigolactone regulation of the root system and the antagonism and interplay with other hormones.
\end{abstract}

Keywords Adventitious roots · Lateral roots ·

Nodulation $\cdot$ Root architecture $\cdot$ Root hairs

The first authors, A. Rasmussen and S. Depuydt, and the last authors, S. Goormachtig and D. Geelen, contributed equally to this work.

A. Rasmussen $\cdot$ D. Geelen

Department of Plant Production, Faculty of Bioscience

Engineering, Ghent University, 9000 Ghent, Belgium

\section{A. Rasmussen}

Division of Plant and Crop Sciences, School of Biosciences,

The University of Nottingham, Sutton Bonington,

Leicestershire LE12 5RD, UK

S. Depuydt $\cdot$ S. Goormachtig $(\bowtie)$

Department of Plant Systems Biology, VIB, Technologiepark

927, 9052 Ghent, Belgium

e-mail: sofie.goormachtig@psb.vib-ugent.be

S. Depuydt $\cdot$ S. Goormachtig

Department of Plant Biotechnology and Bioinformatics,

Ghent University, 9052 Ghent, Belgium

\author{
Abbreviations \\ ABC ATP-binding cassette \\ Afb Auxin signaling f-box \\ ARF Auxin response factor \\ CCD Carotenoid cleavage dioxygenase \\ d dwarf \\ dad Decreased apical dominance \\ max More axillary growth \\ NPA 1- $N$-Naphthylphthalamic acid \\ $\mathrm{N} \quad$ Nitrogen \\ Pi Phosphate \\ PIN PIN FORMED \\ rms ramosus \\ SLR SOLITARYROOT \\ TIBA 2,3,5-Triiodobenzoic acid \\ TIR1 Transport inhibitor response 1
}

\section{Introduction}

Within the rhizosphere, plants interact both positively and negatively with a wide range of organisms, including other plants. Hence, diverse regulatory signals, including plant-derived chemicals, are present in the soil to translate a wealth of information that ultimately leads to growth responses of the interacting partners. An important interaction, which proved crucial for land plant evolution, is the symbiosis with mycorrhizal fungi belonging to the Glomeromycota (Bonfante and Genre 2010). These fungi are obligate heterotrophs that supply the plant with nutrients [mainly phosphate $(\mathrm{Pi})$ and nitrogen $(\mathrm{N})$ ] and, in return, the plant delivers photosynthetic assimilates to the fungi. In addition, mycorrhizal fungi can provide other benefits, such as increased water absorption and root protection from soil heavy metals (Garg and Singla 2011; 
Porcel et al. 2012). An important group of sesquiterpene lactones, commonly named strigolactones, is responsible for stimulating this relationship particularly under nutrient-deficient conditions (Akiyama et al. 2005). More recently in evolution, parasitic plants, such as Striga sp., have co-opted strigolactones as a germination signal, thus indicating proximity to host roots. It is from this interaction that strigolactones derive their name. Many parasitic plants are also obligate heterotrophs and, instead of being beneficial, they drain their hosts of nutrient and energy reserves. These parasitic plants cause extensive yield reductions in agricultural cropping systems across Africa, India and the Middle East (Akiyama and Hayashi 2006; Humphrey and Beale 2006).

Strigolactones have been found across different species and groups of higher plants including rice, petunia, pea, and Arabidopsis (Xie et al. 2010). Recent analysis suggests that strigolactones evolved to control rhizoid elongation because liverworts and Charales produce and respond to strigolactones (Proust et al. 2011; Delaux et al. 2012). In moss, strigolactones control developmental and ecophysiological processes too, in a manner that is reminiscent of quorum-sensing in bacteria (Proust et al. 2011). It is hypothesized that strigolactones were recruited for their role in stimulating mycorrhizal association and maintained for modulating more complex plant architectures (Delaux et al. 2012).

Strigolactones regulate developmental programs and modulate plant architecture through the fine-tuning of shoot branching and root growth (Stirnberg et al. 2007; GomezRoldan et al. 2008; Umehara et al. 2008; Ruyter-Spira et al. 2011). In addition, strigolactones and analogous chemicals (i.e., karrikins) regulate seed germination as part of a light-dependent process (Tsuchiya et al. 2010; Nelson et al. 2011). Because of their role in plant growth and development, strigolactones are considered an additional class of hormones besides the already well-established hormone groups.

A collection of shoot-branching mutants, defective in either strigolactone synthesis or perception [more axillary growth (max) in Arabidopsis, dwarf $(d)$ in rice, decreased apical dominance ( $\mathrm{dad}$ ) in petunia and ramosus (rms) in pea], were instrumental in unraveling the biosynthesis, perception and transport of strigolactones (Fig. 1) (reviewed in Beveridge and Kyozuka 2010). In short, the first step of strigolactone biosynthesis involves the conversion of all-trans- $\beta$-carotene to 9 -cis- $\beta$-carotene that is mediated by D27 (Waters et al. 2012). Next, plastidic carotenoid cleavage dioxygenases 7 and 8 (CCD7 and CCD8 encoded, respectively, by MAX3 and MAX4 in Arabidopsis; Fig. 1) produce carlactone (Alder et al. 2012). Carlactone (or another derived intermediate) is then transported to the cytosol where it is possibly converted to strigolactones by MAXI that encodes a cytoplasmic cytochrome P450 enzyme (Booker et al. 2005; Waters et al. 2012).

Responses to strigolactones require an F-Box leucinerich repeat protein encoded by MAX2/RMS4/D3 and possibly also an $\alpha / \beta$-hydrolase (Gomez-Roldan et al. 2008; Umehara et al. 2008; Arite et al. 2009; Liu et al. 2009; Hamiaux et al. 2012). Based on very recent findings in petunia, the $\alpha / \beta$-hydrolase, encoded by $D A D 2$, hydrolyses GR24 and binds to the MAX2 protein during catalysis, which then leads to an SCF-mediated signal transduction pathway (Hamiaux et al. 2012). Furthermore, the proposed function of DAD2 as a strigolactone receptor is supported by a computationally based structure analysis (Gaiji et al. 2012).

Historically strigolactones were known to be synthesized in both roots and stems and to be transported upwards in the xylem (Kohlen et al. 2011). This is in agreement with measurements of Yoneyama et al. (2007), in which higher levels of strigolactones were found in roots as compared to other tissues. Little is known about what controls the level of strigolactone synthesis, although light quality and intensity could be the determining factors (Koltai et al. 2011). More recently in petunia, an ATPbinding cassette $(\mathrm{ABC})$ transporter PDR1 has been found to play a key role. $p d r 1$ mutants display reduced symbiotic interactions because they are defective in strigolactone exudation, and have an enhanced branching phenotype, like other strigolactone mutants (Kretzschmar et al. 2012). It will be interesting to discover whether and how this transporter exactly acts to control root to shoot distribution of strigolactones.

Despite originally being discovered in plant-related soil signaling, strigolactone regulation of belowground plant architecture had received very little attention. Yet, early studies already reported that strigolactone shoot branching mutants in pea and petunia had reduced root biomass (Beveridge et al. 1996; Snowden et al. 2005; Drummond et al. 2009) whereas the dry weight of other strigolactone mutants did not differ from the wild type (Beveridge et al. 1997a; Morris et al. 2001). In petunia strigolactone mutants, no obvious change in root structure associated with the observed reduction in root mass (Snowden et al. 2005). More detailed analyses of root developmental processes, using strigolactone biosynthesis and signaling mutants in addition to exogenous applications, now show that strigolactones have various roles in regulating the root architecture.

This review will give an overview of the literature regarding the biological activity of strigolactones during root development. Adventitious root formation, primary root growth, lateral root formation, root hair growth and nodulation are all aspects of the root system regulated by 
Fig. 1 Model for strigolactone biosynthesis and perception.

Biosynthesis of strigolactones starts by the sequential cleavage of a carotene precursor (all-trans- $\beta$-carotene) by D27 and then by CCD action in the plastids after which a mobile intermediate, presumably carlactone, moves to the cytosol where the final strigolactone molecules are formed. They are transported upwards in the xylem, and exuded via the PDR1 protein into the rhizosphere and possibly to neighboring cells. The strigolactone signal is perceived by the F-box protein MAX2 and also D14 has been shown to play a role in perception of this class of phytohormones in petunia species. $C C D$ carotenoid cleavage dioxygenase, $P D R$ pleiotropic drug resistance, $S C F$ complex, Skp Cullin, F-box-containing complex

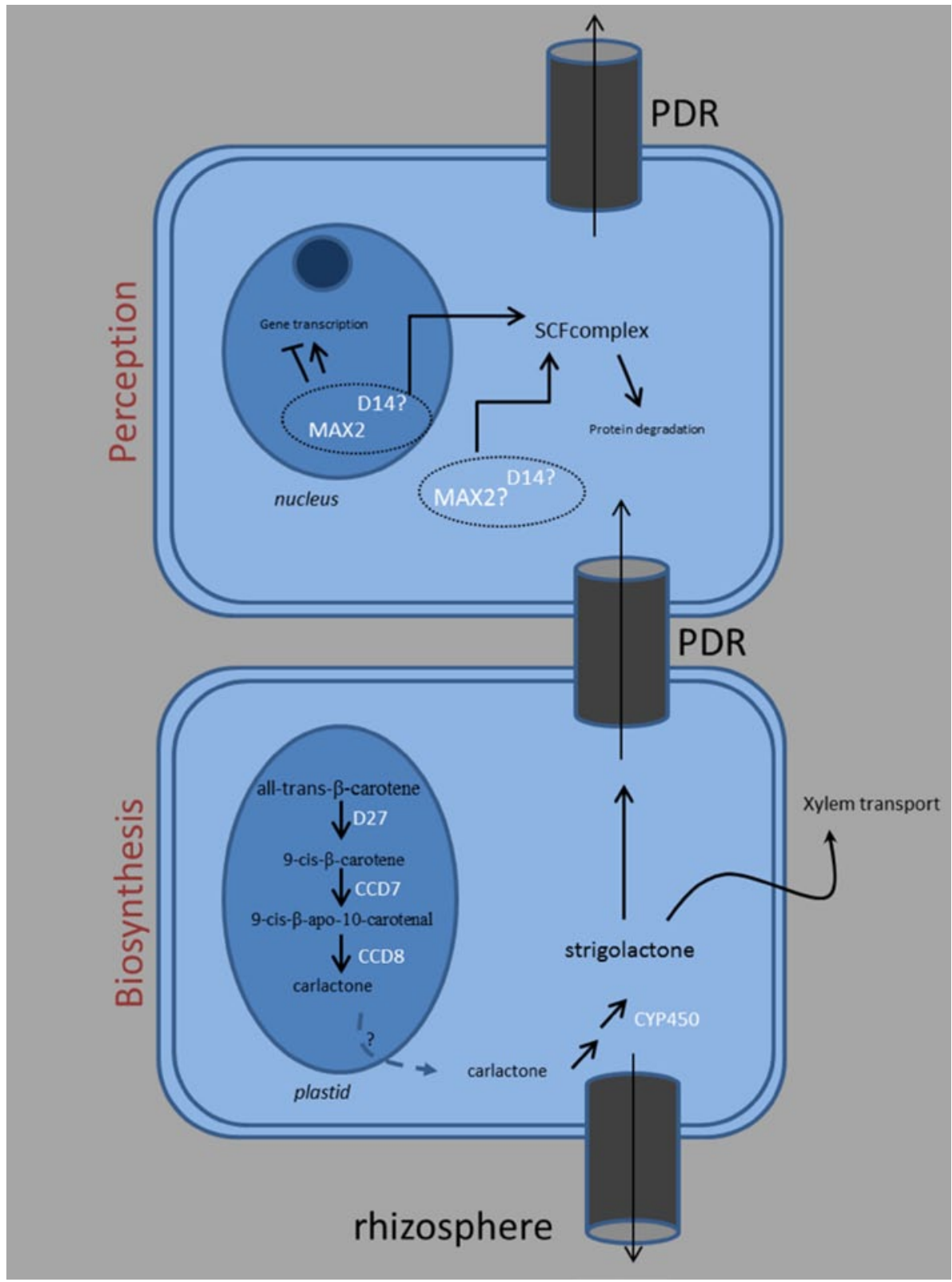

strigolactones. As various established hormones and nutrient conditions influence these processes, we will also discuss their interactions with strigolactones.

\section{Strigolactones inhibit adventitious root formation}

Adventitious roots are defined as roots from non-root tissues, such as stems or leaves, and, as such, can be considered as aerial organs. They are important for all industries relying on clonal propagation including forestry and horticulture. In monocotyledonous species, the early root system consists of a seminal root (primary and short lateral roots) and is replaced by crown roots. Because these roots emerge from stem tissue rather than roots, they are by definition adventitious.

Adventitious roots can be formed from any non-root tissue and, depending on their location, different initiation events have been reported. Adventitious roots forming from Arabidopsis hypocotyls initiate from a cell layer proximate to the central cylinder and in this regard show similarity to the lateral roots that emerge from pericycle founder cells (Casimiro et al. 2001). In many important crop species (including rice, maize and wheat), adventitious roots are formed via vascular cambial cell divisions whereby phloem initials start to divide to produce a primordium (Naija et al. 2009) allowing regeneration of stem cuttings. Under some experimental conditions, cambial cells divide to form 
callus in which tracheids differentiate, elongate and eventually form the center of a complete root primordium (Bollmark et al. 1988; Lorbiecke and Sauter 1999; Rasmussen and Hunt 2010).

One of the most striking effects of strigolactones on the general root structure is the inhibition of adventitious root initiation (Rasmussen et al. 2012). This phenotype has been observed in stem cuttings of pea (Fig. 2) (Rasmussen et al. 2012) and tomato (Kohlen et al. 2012) and etiolated hypocotyls of Arabidopsis (Rasmussen et al. 2012) and as well as in lower stems of intact petunia lacking endogenous strigolactones (Napoli 1996). Exogenous strigolactone treatments inhibited adventitious rooting in a MAX2/RMS4-dependent manner (Kohlen et al. 2012; Rasmussen et al. 2012) and biosynthesis mutants from pea ( $r m s 5)$ but not from Arabidopsis ( $\max 4$ ) were more sensitive to exogenous GR24 application than the wild type (Rasmussen et al. 2012). In stem cuttings, root primordia typically emerge from cambial cell division activity, a stem cell-like cell layer that is required for vascular bundle formation and in addition, secondary growth. Whereas strigolactones may reduce the cambial activity required for adventitious root initiation, they conversely stimulate secondary growth by promoting cambial cell divisions independent or downstream from strigolactone-controlled auxin accumulation (Agusti et al. 2011). These apparently opposing actions in cambial regulation suggest that the role of strigolactones depends on the physiological status of the plant, whereby wound-induced adventitious rooting on cuttings or light-induced Arabidopsis hypocotyls cambium activity is suppressed and stimulated in stems and roots of maturing plants.

Interestingly, the number of crown roots formed by wildtype rice plants was similar to that formed by strigolactone biosynthesis mutants $d 10, d 14, d 17, d 27$ and a perception mutant $d 3$ (Arite et al. 2012). However, impairment of the strigolactone synthesis and perception caused a reduction in rice crown root length, suggesting that strigolactones may regulate adventitious root development differently in monocots and dicots. It is also possible that not all adventitious roots are in fact physiologically identical and crown roots may differ from the adventitious roots appearing on non-root tissues in dicotyledonous plants. Further research on this difference between monocot and dicot adventitious (crown) roots is required to determine whether or not all adventitious roots are similar enough to be compared. This will have ramifications for both clonal propagation and for
Fig. 2 Regulation of the root system by strigolactones, cytokinin (CK), ethylene, auxin and phosphate deficiency. Solid lines represent known interactions while dashed lines represent putative interactions. Arrows symbolize promotion and flat-ended lines inhibition. Ethylene and CK can both also inhibit auxin transport (red vertical lines) but it is unknown whether their regulation of adventitious or lateral roots occurs directly or via changes in auxin transport. Phosphate deficiency increases strigolactone production, but for primary root length, phosphate deficiency and strigolactones act independently and for lateral root formation phosphate-deficient increase in auxin sensitivity lies downstream of a strigolactone-induced inhibition of polar auxin transport. The link between ethylene and strigolactones has only been studied for root hair length

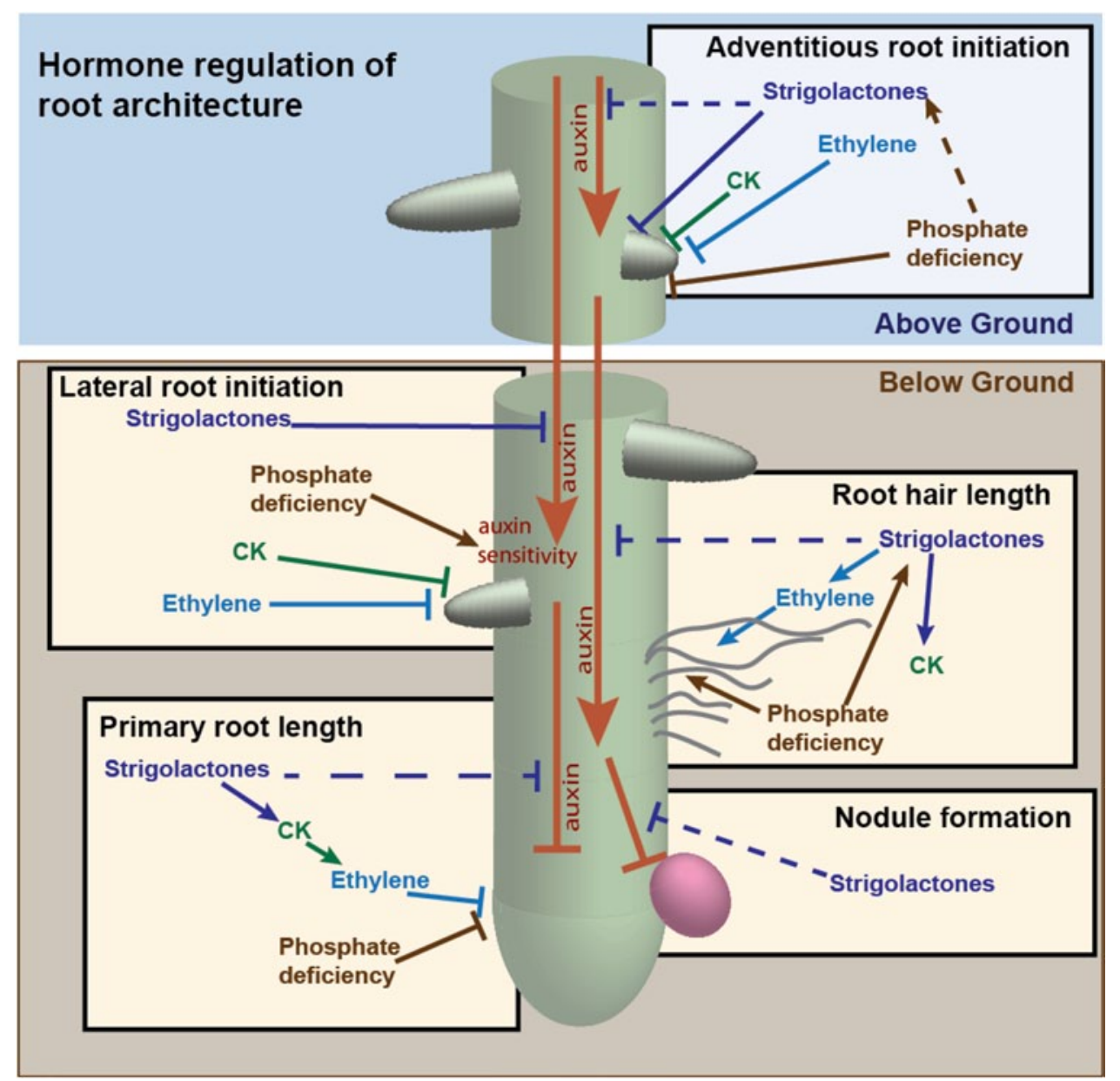


adapting root systems of monocot crops for specific soil environments.

A key question is how strigolactones regulate adventitious root development. Strigolactone mutants have reduced cytokinin levels (Beveridge et al. 1997b; Zhang et al. 2010). As cytokinin is known to be a potent inhibitor of adventitious root formation (Bollmark and Eliasson 1986; De Klerk et al. 2001), cytokinin levels and signaling have been analyzed in Arabidopsis. Strigolactone synthesis and signaling mutants all responded to exogenous cytokinin, and vice versa; both the triple ipt1,5,7 cytokinin synthesis and the double ahk3,4 cytokinin signaling mutants responded to exogenous strigolactones (Rasmussen et al. 2012). Together these findings demonstrate that the increased adventitious rooting in strigolactone mutants is a result of both low strigolactone and low cytokinin levels, independently controlling adventitious rooting (Bollmark and Eliasson 1986; De Klerk et al. 2001; Rasmussen et al. 2012).

In contrast to the inhibitory role of cytokinin, auxin promotes adventitious root formation (Cooper 1936; Boerjan et al. 1995; Delarue et al. 1998). Auxin application was also shown to promote the expression of strigolactone synthesis genes in plant stems (Foo et al. 2005; Hayward et al. 2009), suggesting that auxin should reduce adventitious rooting. However, auxin-induced strigolactone signaling is not the key process regulating adventitious rooting, because both auxin and strigolactone mutants respond to strigolactone and auxin treatments, respectively (Rasmussen et al. 2012). The levels of auxin were found to be higher at the base of tomato stem cuttings (Kohlen et al. 2012) and auxin and strigolactones applied simultaneously to reduce rooting when compared to auxin treatments alone (Kohlen et al. 2012; Rasmussen et al. 2012). Together these results could support several hypotheses. The first is that strigolactone signaling affects the sensitivity of tissues to auxin. The second possibility is that strigolactones may reduce the amount of locally available free auxin, which can then induce adventitious rooting (Kohlen et al. 2012; Rasmussen et al. 2012).

In the regulation of root hairs, it has been demonstrated that strigolactones interact with ethylene (Kapulnik et al. 2011b). So far, there is no direct evidence for a link between strigolactone and ethylene in regulating adventitious rooting; however, ethylene is known to regulate adventitious roots in different ways depending on the conditions. For instance, the activity is strongly dependent on the concentration because at high auxin levels ethylene is inhibitory (De Klerk and Hanecakova 2008; Kilkenny et al. 2012), whereas at low auxin levels ethylene promotes adventitious rooting (De Klerk and Hanecakova 2008). More research is required to determine whether ethylene, strigolactones and auxin interact to regulate adventitious rooting.
Low Pi conditions upregulate strigolactone production, presumably to increase colonization by mycorrhizal fungi (Yoneyama et al. 2012). In this respect it could be expected that low Pi conditions might reduce adventitious root formation as a result of high endogenous strigolactone levels. Indeed, in maize, low Pi has been linked to reduced adventitious rooting (Pellerin et al. 2000; Kume et al. 2006). The study of Pellerin et al. (2000) revealed that emergence of adventitious roots (called crown roots in maize) was delayed under Pi-deficient conditions. Kume et al. (2006) exposed individual maize plants to heterogeneous soil patches and found that adventitious roots were preferentially formed on the sides of the stem exposed to Pi-enriched patches. As the strigolactone levels were not measured in these studies, it remains possible that these phenotypes result from other hormone interactions.

\section{Strigolactones increase primary root length}

Several recent reports show that the strigolactone production and response mutants of rice, maize and pea have shorter primary roots than the wild type (Fig. 2) (Arite et al. 2012; Guan et al. 2012; Kretzschmar et al. 2012). In support of the strigolactone-promoting primary root growth, Ruyter-Spira et al. (2011) demonstrated that when grown without sucrose, GR24 applications trigger root growth in a MAX2-dependent manner. They also measured the number of cells in the meristem and showed that GR24 increased the number of cells in the meristem, possibly explaining the increase in root length. However, $0.5 \%$ sucrose was used for counting the meristem cell numbers and under these conditions primary root length was inhibited by GR24 independently of MAX2. Shinohara et al. (2013) confirmed this MAX2-dependent root growth inhibition by GR24 levels between 3 and $30 \mu \mathrm{M}$. Another study (Dubrovsky et al. 2009) found cortical cells in the primary root of $\max 4$ to be shorter than those of the wild type, but root length was not mentioned. Further support for a promoting role for strigolactones in primary root length comes from a study where GR24 was shown to increase root length in tomato (Koltai et al. 2010) but only after root length inhibition by auxin and only significant at very high concentrations. This study also demonstrated that GR24 could increase cortical cell length in the presence of auxin, while $C Y C B 1$ expression (indicative of cell division rates) was inhibited by GR24. In contrast to the study by Ruyter-Spira et al. (2011), the findings of Koltai et al. (2010) suggest that the longer primary root results from enhanced cell elongation rather than cell division. These contrasting reports demonstrate the need for further research to understand how strigolactones regulate cell division or elongation. 
In contrast to lateral root formation, Pi-deficient inhibition of root growth occurs independently of strigolactone signaling, as evidenced by Pi-deficient inhibition of primary root growth in both max 2 and wild type (Yoneyama et al. 2007; Jain et al. 2007; Mayzlish-Gati et al. 2012).

Strigolactones may regulate local levels of auxin either by changing auxin biosynthesis or polar auxin transport. Some studies have demonstrated that in the strigolactone mutants the auxin levels were higher (Beveridge et al. 1997b; Bennett et al. 2006) whereas others that the mutants and the wild type did not differ (Beveridge et al. 1996; Morris et al. 2001; Arite et al. 2009). There is also support for a role of strigolactones in reducing polar auxin transport both by means of radiolabeled auxins and PIN efflux marker lines, although contradictory results concerning subcellular relocalisation of PINs in root tips have been reported in different experiments and when the root and shoot systems were compared (Beveridge et al. 2000; Bennett et al. 2006; Lazar and Goodman 2006; Lin et al. 2009; Crawford et al. 2010; Shinohara et al. 2013). Recently, strigolactone action in stems was found to trigger PIN1 depletion from the plasma membrane of xylem parenchyma cells and depended on clathrin-mediated membrane trafficking (Shinohara et al. 2013). This regulation of auxin may explain some of the strigolactone-related phenotypes described here.

Increased auxin transport in the strigolactone mutants results in more auxin reaching the root. Because auxin inhibits root growth, this may explain the reduced root length in these plants and the enhanced root length in plants treated exogenously with strigolactone. However, this is speculative and investigations into this interaction including cell-level analyses are still required. Interestingly, the Pi-deficient decrease in root length mentioned above has been proposed to occur independently of auxin (Jain et al. 2007), whereas in contrast, the interaction between $\mathrm{Pi}$ and auxin appears to be involved in the regulation of lateral root development (Jain et al. 2007) (see below).

\section{Strigolactone regulation of lateral root formation depends on the nutrient environment}

Lateral roots originate from the pericycle either adjacent to xylem pole cells (in dicotyledons) or phloem pole cells (in monocotyledons), although under exceptional circumstances lateral roots can also emerge from the endodermis (Casero et al. 1995). The first steps in lateral root formation are anticlinal divisions of pericycle cells that were arrested in the G2 phase of the cell cycle (Beeckman et al. 2001). As such they become lateral root founder cells, which can become activated to initiate the development of a lateral root primordium containing the same cell types as a mature root (Malamy and Benfey 1997).
A crucial step for founder cell specification is the accumulation of auxin. This is evidenced in auxin receptor mutants (tirlafb2afb3) that show a drastic reduction in lateral root formation (Dharmasiri et al. 2005; Pérez-Torres et al. 2008) and in several mutants perturbed in $A U X / I A A-$ and $A R F$-mediated signaling that display severe defects in lateral root organogenesis, or block it completely [in the case of stabilized SOLITARY ROOT(SLR)/IAA14 or loss of $A R F 7$ and $A R F 19$ (Vanneste et al. 2005)]. Local accumulation of auxin in root pericycle cells is ensured by PINdependent auxin recirculation inside the root tip (Casimiro et al. 2001), a process that is very well described elsewhere and falls beyond the scope of this review. Following lateral root initiation, organogenesis of lateral root primordia is achieved by highly coordinated cell division and differentiation patterns (Benková and Bielach 2010).

Strigolactones affect auxin fluxes (see above) (Bennett et al. 2006; Brewer et al. 2009; Crawford et al. 2010; Ruyter-Spira et al. 2011; Shinohara et al. 2013), so it was expected that strigolactones may also have an effect on lateral root formation. Thus far, two reports describe a negative influence of strigolactones on lateral root density in a MAX2-dependent manner (Fig. 2) (Kapulnik et al. 2011a; Ruyter-Spira et al. 2011). However, an increased lateral root density in the max mutants under control conditions was observed only sometimes (Kapulnik et al. 2011a), or only under Pi-limiting conditions (Ruyter-Spira et al. 2011).

Both reports on strigolactone regulation of lateral root formation presented different conclusions regarding the underlying cause for the inhibition (Kapulnik et al. 2011a; Ruyter-Spira et al. 2011). Ruyter-Spira et al. (2011) found that GR24 treatment prevented the transition of lateral root primordia from stage $\mathrm{V}$ to stage VI in both wild type and strigolactone biosynthesis mutants (except max4). In contrast, Kapulnik et al. (2011a) suggest that the initiation of lateral roots was affected rather than the elongation or outgrowth, but this was highly dependent on the applied concentration of strigolactones. Although it is difficult to reconcile these two contrasting reports, part of the mechanism for strigolactone regulation of lateral root formation may result from changes in auxin transport capacity (reduction of PIN1 expression) (Ruyter-Spira et al. 2011). By applying enough auxin to the growing seedlings (restoring PIN1 expression to normal levels and patterns), the inhibitory effect of strigolactones on lateral root development was counteracted (Ruyter-Spira et al. 2011). However, no data for the lateral root density were presented for auxin or for auxin together with strigolactones, making conclusions difficult. Despite this, these findings suggest that decreased shoot-derived auxin levels (at least partially) mediate the GR24-induced suppression of lateral root primordium outgrowth (Ruyter-Spira et al. 2011). 
The interaction between strigolactones and cytokinins has been found to be independent in the regulation of adventitious root formation (Rasmussen et al. 2012). This interaction has not yet been studied for lateral roots, but, similar to adventitious rooting, cytokinins are negative regulators of lateral roots (Laplaze et al. 2007). Cytokinins arrest the pericycle founder cells in the G2-to-M transition, preventing their outgrowth into mature lateral roots and the correct establishment of auxin maxima as well by interfering with PIN transporters affecting lateral root primordia patterning (Laplaze et al. 2007). It will be interesting in future studies to determine how/whether strigolactones and cytokinins interact to regulate lateral root development because the strigolactone mutants also contain reduced cytokinin levels. A very novel and exciting observation in that respect comes from a recent study by Koren et al. (2013) in which they show that strigolactone signaling in the endodermis is sufficient for mediating lateral root responses towards exogenous GR24. Moreover, shy2 loss-of-function mutants were found to be insensitive to GR24 treatment for both lateral root formation and cell meristem number, revealing a role for this regulator of root meristem size not only during the cytokinin and auxin interplay (Dello Ioio et al. 2008), but also during strigolactone action in shaping the root system.

High levels of inorganic Pi suppress lateral root elongation and decrease lateral root density, but stimulate primary root elongation. In contrast, under low levels of Pi, lateral root initiation and elongation are favored over primary root growth (Williamson et al. 2001) while strigolactone production increases (Yoneyama et al. 2007). Importantly, the action of strigolactones on lateral root formation/initiation depends on the Pi status of the plant. Under Pi-sufficient conditions (including the studies described above) strigolactones inhibit lateral root formation, whereas under Pi-limiting conditions the converse is true (Ruyter-Spira et al. 2011). This effect could also correlate with changes in auxin responses in the root meristem (López-Bucio et al. 2003). Indeed, the increase in auxin sensitivity following $\mathrm{Pi}$ deprivation results in a loss of the inhibitory effect of strigolactones on lateral root development (Ruyter-Spira et al. 2011). This could be consistent with the Pi-deficient increase in auxin sensitivity lying downstream of the strigolactone-inhibited auxin transport in regulating lateral root formation. These findings lead to the hypothesis that strigolactones might translate environmental signals into appropriate growth cues for the root system architecture although solid evidence still needs to be provided (RuyterSpira et al. 2011; Ruyter-Spira and Bouwmeester 2012).

\section{Root hair length is enhanced by strigolactones}

Root hairs not only facilitate the absorption of water and nutrients due to a large enhancement of root surface area
(Jones and Dolan 2012), but also help in establishing the symbiotic interaction between rhizobia and leguminous plants. The formation of a root hair, via outgrowth of epidermal cells (or trichoblasts), is divided into specific stages (cell specification, root hair initiation, tip growth, and maturation) (Pei et al. 2012), and the decision to become a hair cell or not, requires specific positional information from the underlying cortical cell layer.

It has been well described that root hair elongation results from increased auxin levels in the epidermal cells and AUX1-dependent transport through non-hair epidermal cells (Pitts et al. 1998; Jones et al. 2009). Ethylene has also been implied in the root hair elongation process, possibly via cross-talk with auxin (Pitts et al. 1998; Kapulnik et al. 2011b), because at low levels, ethylene promotes auxin biosynthesis and transport in the root (Stepanova et al. 2007; Swarup et al. 2007).

Strigolactones are implicated in root hair elongation as well (Fig. 2). Arabidopsis strigolactone mutants had shorter root hairs than the wild type and a significant increase in root hair length was observed upon exogenous GR24 treatment in wild-type and $\max 3$ and $\max 4$ mutant plants, whereas addition to $\max 2$ roots had no effect (Kapulnik et al. 2011a). As observed in lateral root development, this MAX2-dependent root hair length phenotype is concentration-dependent and at high concentrations even the $\max 2$ mutant responds to GR24. In contrast, it was found that GR24 inhibited root hair elongation in tomato; however, this only occurred at non-physiological concentrations and was not tested at lower concentrations (Koltai et al. 2010). At these high concentrations they also found that auxin could not rescue the phenotype. Instead the auxin transport inhibitor 1-N-naphthylphthalamic acid (NPA) was able to enhance the root hair length, suggesting that high levels of strigolactones enhance auxin efflux in tomato (Koltai et al. 2010).

Further analyses of the relationship between ethylene, auxin and strigolactones demonstrate that ethylene biosynthesis is required for strigolactone-mediated root hair elongation (Kapulnik et al. 2011b). In contrast, auxin was not required for strigolactone-enhanced root hair elongation, because the tirl auxin response mutant was still responsive to exogenous GR24 (Kapulnik et al. 2011b). Despite this, auxin signaling enhances the strigolactone-induced root hair response, suggesting a convergence of the strigolactone and auxin hormonal pathways.

Nutrient conditions also affect root hair formation. For example low $\mathrm{Pi}, \mathrm{N}$ and iron all induce root hair formation (Gahoonia et al. 2001; López-Gucio et al. 2003). However, little has been reported on the effect of nutrient deficiency and root hair length, which seems to be the aspect of root hair development most affected by strigolactones. However, it has been suggested that the root hair response to low Pi 
is mediated by strigolactones and could be correlated with transcriptional activation of TIRl, thus complementing the experimental evidence that auxin is sufficient to compensate for the reduced $\max 2$ responses (Mayzlish-Gati et al. 2012).

\section{More root organs: nodule formation is enhanced by exogenous strigolactones}

Leguminous plants have the peculiar ability to engage in a symbiotic interaction with rhizobia resulting in the development of new root organs, the nodules, inside of which the bacteria fix atmospheric $\mathrm{N}$ for the plant. Nodule development is a complex program that involves bacterial infection and re-initiation of cell division in the cortex.

The nodulation process is initiated after the plant perceives Nod factors, i.e., lipochitooligosaccharides that are produced by rhizobia. With the discovery of strigolactones being both a rhizosphere signal and an endogenous plant hormone, the question arose whether strigolactones play a role in nodulation either as an endogenous plant hormone or a rhizosphere signal. It is well established that the nodulation process initiates when the bacteria perceive plant secondary metabolites, essentially flavonoid compounds, via the NodD transcriptional activator (Oldroyd et al. 2011). Perception of the proper flavonoid compounds activates the rhizobial nod genes, resulting in the production and secretion of Nod factors (Oldroyd et al. 2011). However, addition of GR24 to rhizobial cell cultures could not induce nod gene expression, indicating that strigolactones do not contribute to the initiation of Nod factor production by the rhizobia (Moscatiello et al. 2010; Soto et al. 2010). Hence, in the rhizosphere, strigolactones seem to solely act as a recognition signal for arbuscular myccorhizal fungi and parasitic plants and not for rhizobia.

Still, exogenous addition of GR24 was found to positively influence the number of nodules in Medicago sativa (Fig. 2) (Soto et al. 2010), supporting the role of strigolactones in regulating organ development. In agreement, the pea mutant rmsl was shown to carry less nodules per gram weight compared to control plants and this effect was complemented by exogenous GR24 (Foo and Davies 2011). These data are in agreement with a positive role of endogenous strigolactones in nodulation. Interestingly, rms 4 mutants affected in strigolactone response carried more nodules than the wild type, which is the opposite phenotype to rmsl (Foo et al. 2013). This discrepancy demonstrates that there is still much to be discovered about strigolactone signaling and it supports the hypothesis that the F-box protein is involved in signaling processes other than just RMS1-produced strigolactones (Waters et al. 2011).

The M. truncatula nsp 1 and nsp 2 nodulation mutants, affected in GRAS-type transcription factors within the Nod factor signaling pathway, are seriously hampered in strigolactone biosynthesis and D27 expression in non-symbiotic conditions (Liu et al. 2011). Those mutants are strongly affected in nodulation and $n s p 2$ and $n s p 1 / n s p 2$ mutants also show a slight decrease in arbuscular mycorrhization (Liu et al. 2011; Maillet et al. 2011). These data indicate that the low level of strigolactones might contribute to the nodulation phenotype. However, the $n s p l$ and $n s p 2$ mutants have a much stronger nodulation defect compared to the rms 1 mutant in pea, which is also hampered in strigolactone production. In addition, the decrease in $d 27$ expression was not observed in mutants affected in Nod factor signaling components that act upstream of NSP1 and NSP2 to control nodulation (Liu et al. 2011). Hence, whether the observed decrease in strigolactone production contributes to the lack of nodulation observed in $n s p 1$ and $n s p 2$ is currently not known but should be tested to unravel how and whether the observations in the $n s p 1$ and $n s p 2$ mutants are linked to the positive role of strigolactones in nodulation. Alternatively, these GRAS-type transcription factors might control different groups of genes to regulate nodulation or arbuscular mycorrhization.

Strigolactones might influence the auxin transport and signaling cascades that control nodule establishment. Indeed, application of polar auxin transport inhibitors such as NPA and 2,3,5-triiodobenzoic acid (TIBA) can induce spontaneous nodules in some legumes (Allen et al. 1953; Hirsch et al. 1989; Wu et al. 1996; Rightmyer and Long 2011). It has been shown that some genes transcriptionally controlled by both nodulation and auxin transport inhibitors might be important for nodule initiation (Rightmyer and Long 2011). In agreement, physiological experiments in vetch, white clover and $M$. truncatula have revealed that at the nodule initiation site, a local inhibition of polar auxin transport takes place that might allow auxin accumulation at the site of nodule primordia development (Mathesius et al. 1998; Boot et al. 1999; Prayitno et al. 2006; van Noorden et al. 2006). Besides a local influence on auxin transport, the development of the first nodules reduces the long-distance shoot-to-root transport of auxins (van Noorden et al. 2006) that might be fundamental to control nodule numbers (van Noorden et al. 2006). Hence, it will be interesting to test whether strigolactones impinge on one of these processes to control nodule development by carefully analyzing strigolactone action in relation to auxin distribution and PIN expression.

\section{Are all strigolactone effects related to auxin?}

In the shoot there are clear links between strigolactone and auxin (Bennett et al. 2006; Brewer et al. 2009; Crawford et al. 2010; Ruyter-Spira et al. 2011; Shinohara et al. 2013). In particular, it seems likely that strigolactones regulate local auxin levels. In belowground phenotypes it is also 
possible that strigolactones may be responsible for reducing local auxin levels. As indicated above, this may explain the inhibitory effect of strigolactones on adventitious rooting, lateral root formation, root hair length and nodulation while promoting primary root length.

The possibility that strigolactones may reduce auxin biosynthesis in belowground tissues is supported by DR5:GUS results (Ruyter-Spira et al. 2011). Auxin is also regulated by positive feedback loops between biosynthesis and transport, so the higher levels of auxin biosynthesis may cause the higher levels of transporter proteins observed in primary roots (Ruyter-Spira et al. 2011) and also the higher levels of auxin measured at the bases of cuttings from tomato strigolactone mutants (Kohlen et al. 2012). Opposing evidence for this theory is that the strigolactone mutants of pea do not necessarily have a higher auxin content than that of the wild type (Beveridge et al. 1996; Morris et al. 2001). Additional proof against strigolactone directly regulating auxin biosynthesis is found in root hair lengths, where exogenous treatments with auxins using the PIN efflux pathway were unable to restore the phenotype (Koltai et al. 2010), suggesting a more direct interaction with the PIN pathway.

Another possibility is that strigolactones directly affect the PIN efflux pathway, which is supported by recent evidence in Arabidopsis stems where it was shown that strigolactone treatment triggers PIN1 depletion from the plasma membrane (Shinohara et al. 2013). Also in root hair length studies direct interaction with polar auxin transport was suggested (Koltai et al. 2010). The reduction in PIN expression levels with strigolactones (Ruyter-Spira et al. 2011) also provides support for this theory. The positive feedback loops can also explain the changes in DR5 levels, as an increase in transport away from the biosynthesis locations causes an increase in biosynthesis (Beveridge et al. 1997b; Bennett et al. 2006). An increase in polar auxin transport would also cause the increased auxin levels found at the base of strigolactone mutant cuttings (Kohlen et al. 2012) and the higher DR5 levels observed in the primary root (Ruyter-Spira et al. 2011). A reduction in polar auxin transport following GR24 treatment can also explain the findings that exogenous auxin added together with strigolactones has a less pronounced phenotype than when auxin is added alone (Kohlen et al. 2012; Rasmussen et al. 2012), because the polar auxin stream would become a bottleneck, reducing the amount of auxin reaching the base of the cutting. Nevertheless, newer evidence has contradicted the reported down-regulation of PIN proteins upon GR24 treatment (Shinohara et al. 2013). Hence, two distinct models are proposed for auxin-strigolactone interaction in both shoots and roots. The forthcoming research on how strigolactones regulate root system architecture will undoubtedly shed new light on this tight and very interesting interaction between phytohormones.

\section{Conclusion}

It is clear that strigolactones are important regulators of many aspects of the root system architecture. Besides welldescribed effects of strigolactones to increase mycorrhizal infection rates and nodulation, root hair elongation is also induced by strigolactones, whereas adventitious root initiation and lateral rooting is inhibited. When comparing the regulation of these root system components, certain differences appear between the interaction of strigolactones and other root shaping hormones, although all aspects are additionally heavily influenced by nutrient conditions. For instance, clear links between cytokinin and strigolactones are thus far only published for adventitious rooting, whereas ethylene has only been implied during the strigolactone effect on root hair formation. It is possible that the differences in cross-talk account for the pleiotropic effect of strigolactones on different aspects of the root system. Researchers readily explain many of the observed phenotypes by an effect of strigolactones on fine-tuning local auxin levels via changes in auxin transport. However, evidence of a direct effect of strigolactones on any phenotype has not been ruled out, so care must be taken. Also, the differences in responses between different environmental conditions need to be kept in mind for future studies to untangle the signaling networks involved in root system regulation. In addition, because the soil environment is very heterogeneous in terms of nutrients, it will be important to test the effect of strigolactones on root system development under such conditions, which will contribute to our current (incomplete) understanding of how strigolactones affect the belowground organization of the plant. At the molecular level there are also huge gaps in our knowledge on the nature of strigolactone regulation of phenotypes and interactions with other signals. In conclusion, further research is still required to understand the complex signaling networks leading to regulation of the root system, crucial for plant growth.

Acknowledgments A.R. is the recipient of a Marie Curie International Incoming Fellowship and a Newton International Fellowship. S.D. is a postdoctoral researcher of the Research Foundation-Flanders.

Conflict of interest The authors declare that they have no conflict of interest.

\section{References}

Agusti J, Herold S, Schwarz M, Sanchez P, Ljung K, Dun EA, Brewer PB, Beveridge CA, Sieberer T, Sehr EM, Greb T (2011) Strigolactone signaling is required for auxin-dependent stimulation of secondary growth in plants. Proc Natl Acad Sci USA 108:20242-20247 
Akiyama K, Hayashi H (2006) Strigolactones: chemical signals for fungal symbionts and parasitic weeds in plant roots. Ann Bot 97:925-931

Akiyama K, Matsuzaki KI, Hayashi H (2005) Plant sesquiterpenes induce hyphal branching in arbuscular mycorrhizal fungi. Nature 435:824-827

Alder A, Jamil M, Marzorati M, Bruno M, Vermathen M, Bigler P, Ghisla S, Bouwmeester H, Beyer P, Al-Babili S (2012) The path from $\beta$-carotene to carlactone, a strigolactone-like plant hormone. Science 335:1348-1351

Allen EK, Allen ON, Newman AS (1953) Pseudonodulation of leguminous plants induced by 2-bromo-3,5-dichlorobenzoic acid. Am J Bot 40:429-435

Arite T, Umehara M, Ishikawa S, Hanada A, Maekawa M, Yamaguchi S, Kyozuka J (2009) d14, a strigolactone-insensitive mutant of rice, shows an accelerated outgrowth of tillers. Plant Cell Physiol 50:1416-1424

Arite T, Kameoka H, Kyozuka J (2012) Strigolactone positively controls crown root elongation in rice. J Plant Growth Regul 31:165-172

Beeckman T, Burssens S, Inzé D (2001) The peri-cell-cycle in Arabidopsis. J Exp Bot 52:403-411

Benková E, Bielach A (2010) Lateral root organogenesis-from cell to organ. Curr Opin Plant Biol 13:677-683

Bennett T, Sieberer T, Willett B, Booker J, Luschnig C, Leyser O (2006) The Arabidopsis MAX pathway controls shoot branching by regulating auxin transport. Curr Biol 16:553-563

Beveridge CA, Kyozuka J (2010) New genes in the strigolactonerelated shoot branching pathway. Curr Opin Plant Biol 13:34-39

Beveridge CA, Ross JJ, Murfet IC (1996) Branching in pea-action of genes rms3 and rms4. Plant Physiol 110:859-865

Beveridge CA, Murfet IC, Kerhoas L, Sotta B, Miginiac E, Rameau C (1997a) The shoot controls zeatin riboside export from pea roots. Evidence from the branching mutant rms4. Plant $\mathrm{J}$ 11:339-345

Beveridge CA, Symons GM, Murfet IC, Ross JJ, Rameau C (1997b) The rms 1 mutant of pea has elevated indole-3-acetic acid levels and reduced root-sap zeatin riboside content but increased branching controlled by graft-transmissible signal(s). Plant Physiol 115:1251-1258

Beveridge CA, Symons GM, Turnbull CGN (2000) Auxin inhibition of decapitation-induced branching is dependent on grafttransmissible signals regulated by genes $r m s 1$ and $r m s 2$. Plant Physiol 123:689-697

Boerjan W, Cervera M-T, Delarue M, Beeckman T, Dewitte W, Bellini C, Caboche M, Van Onckelen H, Van Montagu M, Inzé D (1995) superroot a recessive mutation in Arabidopsis, confers auxin overproduction. Plant Cell 7:1405-1419

Bollmark M, Eliasson L (1986) Effects of exogenous cytokinins on root formation in pea cuttings. Physiol Plant 68:662-666

Bollmark M, Kubát M, Eliasson L (1988) Variation in endogenous cytokinin content during adventitious root formation in pea cuttings. J Plant Physiol 132:262-265

Bonfante P, Genre A (2010) Mechanisms underlying beneficial plantfungus interactions in mycorrhizal symbiosis. Nat Commun $1: 48$

Booker J, Sieberer T, Wright W, Williamson L, Willett B, Stirnberg P, Turnbull C, Srinivasan M, Goddard P, Leyser O (2005) MAX1 encodes a cytochrome P450 family member that acts downstream of $M A X 3 / 4$ to produce a carotenoid-derived branchinhibiting hormone. Dev Cell 8:443-449

Boot KJM, van Brussel AAN, Tak T, Spaink HP, Kijne JW (1999) Lipochitin oligosaccharides from Rhizobium leguminosarum bv. viciae reduce auxin transport capacity in Vicia sativa subsp. nigra roots. Mol Plant Microbe Interact 12:839-844
Brewer PB, Dun EA, Ferguson BJ, Rameau C, Beveridge CA (2009) Strigolactone acts downstream of auxin to regulate bud outgrowth in pea and Arabidopsis. Plant Physiol 150:482-493

Casero PJ, Casimiro I, Lloret PG (1995) Lateral root initiation by asymmetrical transverse divisions of pericycle cells in four plant species: Raphanus sativus, Helianthus annuus, Zea mays, and Daucus carota. Protoplasma 188:49-58

Casimiro I, Marchant A, Bhalerao RP, Beeckman T, Dhooge S, Swarup R, Graham N, Inzé D, Sandberg G, Casero PJ, Bennett M (2001) Auxin transport promotes Arabidopsis lateral root initiation. Plant Cell 13:843-852

Cooper WC (1936) Transport of root-forming hormone in woody cuttings. Plant Physiol 11:779-793

Crawford S, Shinohara N, Sieberer T, Williamson L, George G, Hepworth J, Müller D, Domagalska MA, Leyser O (2010) Strigolactones enhance competition between shoot branches by dampening auxin transport. Development 137:2905-2913

De Klerk G-J, Hanecakova J (2008) Ethylene and rooting of mung bean cuttings. The role of auxin induced ethylene synthesis and phase-dependent effects. Plant Growth Regul 56:203-209

De Klerk G-J, Hanecakova J, Jasik J (2001) The role of cytokinins in rooting of stem slices cut from apple microcuttings. Plant Biosyst 135:79-84

Delarue M, Prinsen E, Van Onckelen H, Caboche M, Bellini C (1998) Sur2 mutations of Arabidopsis thaliana define a new locus involved in the control of auxin homeostasis. Plant J 14:603-611

Delaux P-M, Xie X, Timme RE, Puech-Pages V, Dunand C, Lecompte E, Delwiche CF, Yoneyama K, Bécard G, Séjalon-Delmas N (2012) Origin of strigolactones in the green lineage. New Phytol 195:857-871

Dello Ioio R, Nakamura K, Moubayidin L, Perilli S, Taniguchi M, Morita MT, Aoyama T, Costantino P, Sabatini S (2008) A genetic framework for the control of cell division and differentiation in the root meristem. Science 322:1380-1384

Dharmasiri N, Dharmasiri S, Estelle M (2005) The F-box protein TIR1 is an auxin receptor. Nature 435:441-445

Drummond RSM, Martínez-Sánchez NM, Janssen BJ, Templeton KR, Simons JL, Quinn BD, Karunairetnam S, Snowden KC (2009) Petunia hybrida CAROTENOID CLEAVAGE DIOXYGENASE7 is involved in the production of negative and positive branching signals in petunia. Plant Physiol 151:1867-1877

Dubrovsky JG, Soukup A, Napsucialy-Mendivil S, Jeknić Z, Ivanchenko MG (2009) The lateral root initiation index: an integrative measure of primordium formation. Ann Bot 103:807-817

Foo E, Davies NW (2011) Strigolactones promote nodulation in pea. Planta 234:1073-1081

Foo E, Buillier E, Goussot M, Foucher F, Rameau C, Beveridge CA (2005) The branching gene RAMOSUS1 mediates interactions among two novel signals and auxin in pea. Plant Cell $17: 464-474$

Foo E, Yoneyama K, Hugill CJ, Quittenden LJ, Reid JB (2013) Strigolactones and the regulation of pea symbioses in response to nitrate and phosphate deficiency. Mol Plant 6:76-87

Gahoonia TS, Nielsen NE, Joshi PA, Jahoor A (2001) A root hairless barley mutant for elucidating genetic of root hairs and phosphorus uptake. Plant Soil 235:211-219

Gaiji N, Cardinale F, Prandi C, Bonfante P, Ranghino G (2012) The computational-based structure of Dwarf14 provides evidence for its role as potential strigolactone receptor in plants. BMC Res Notes 5:307

Garg N, Singla P (2011) Arsenic toxicity in crop plants: physiological effects and tolerance mechanisms. Environ Chem Lett 9:303-321

Gomez-Roldan V, Fermas S, Brewer PB, Puech-Pagès V, Dun EA, Pillot J-P, Letisse F, Matusova R, Danoun S, Portais 
J-C, Bouwmeester H, Bécard G, Beveridge CA, Rameau C, Rochange SF (2008) Strigolactone inhibition of shoot branching. Nature 455:189-194

Guan JC, Koch KE, Suzuki M, Wu S, Latshaw S, Petruff T, Goulet C, Klee HJ, McCarty DR (2012) Diverse roles of strigolactone signaling in maize architecture and the uncoupling of a branching-specific sub-network. Plant Physiol 160:1303-1317

Hamiaux C, Drummond RSM, Janssen BJ, Ledger SE, Cooney JM, Newcomb RD, Snowden KC (2012) DAD2 is an a/b hydrolase likely to be involved in the perception of the plant branching hormone, strigolactone. Curr Biol 22:2032-2036

Hayward A, Stirnberg P, Beveridge CA, Leyser O (2009) Interactions between auxin and strigolactone in shoot branching control. Plant Physiol 151:400-412

Hirsch AM, Bhuvaneswari TV, Torrey JG, Bisseling T (1989) Early nodulin genes are induced in alfalfa root outgrowths elicited by auxin transport inhibitors. Proc Natl Acad Sci USA 86:1244-1248

Humphrey AJ, Beale MH (2006) Strigol: biogenesis and physiological activity. Phytochemistry 67:636-640

Jain A, Poling MD, Karthikeyan AS, Blakeslee JJ, Peer WA, Titapiwatanakun B, Murphy AS, Raghothama KG (2007) Differential effects of sucrose and auxin on localized phosphate deficiencyinduced modulation of different traits of root system architecture in Arabidopsis. Plant Physiol 144:232-247

Jones VAS, Dolan L (2012) The evolution of root hairs and rhizoids. Ann Bot 110:205-212

Jones AR, Kramer EM, Knox K, Swarup R, Bennett MJ, Lazarus CM, Leyser HMO, Grierson CS (2009) Auxin transport through nonhair cells sustains root-hair development. Nat Cell Biol 11:78-84

Kapulnik Y, Delaux P-M, Resnick N, Mayzlish-Gati E, Wininger S, Bhattacharya C, Séjalon-Delmas N, Combier J-P, Bécard G, Belausov E, Beeckman T, Dor E, Hershenhorn J, Koltai H (2011a) Strigolactones affect lateral root formation and roothair elongation in Arabidopsis. Planta 233:209-216

Kapulnik Y, Resnick N, Mayzlish-Gati E, Kaplan Y, Wininger S, Hershenhorn J, Koltai H (2011b) Strigolactones interact with ethylene and auxin in regulating root-hair elongation in Arabidopsis. J Exp Bot 62:2915-2924

Kilkenny AJ, Wallace HM, Walton DA, Adkins MF, Trueman SJ (2012) Improved root formation in Eucalypt cuttings following combined auxin and anti-ethylene treatments. J Plant Sci 7:138-153

Kohlen W, Charnikhova T, Liu Q, Bours R, Domagalska MA, Beguerie S, Verstappen F, Leyser O, Bouwmeester H, RuyterSpira C (2011) Strigolactones are transported through the xylem and play a key role in shoot architectural response to phosphate deficiency in nonarbuscular mycorrhizal host Arabidopsis. Plant Physiol 155:974-987

Kohlen W, Charnikhova T, Lammers M, Pollina T, Tóth P, Haider I, Pozo MJ, de Maagd RA, Ruyter-Spira C, Bouwmeester HJ, López-Ráez JA (2012) The tomato CAROTENOID CLEAVAGE DIOXYGENASE8 (SICCD8) regulates rhizosphere signaling, plant architecture and affects reproductive development through strigolactone biosynthesis. New Phytol 196:535-547

Koltai H, Dor E, Hershenhorn J, Joel DM, Weininger S, Lekalla S, Shealtiel H, Bhattacharya C, Eliahu E, Resnick N, Barg R, Kapulnik Y (2010) Strigolactones' effect on root growth and root-hair elongation may be mediated by auxin-efflux carriers. J Plant Growth Regul 29:129-136

Koltai H, Cohen M, Chesin O, Mayzlish-Gati E, Bécard G, Puech V, Ben Dor B, Resnick N, Wininger S, Kapulnik Y (2011) Light is a positive regulator of strigolactone levels in tomato roots. $\mathrm{J}$ Plant Physiol 168:1993-1996

Koren D, Resnick N, Gati EM, Belausov E, Weininger S, Kapulnik Y, Koltai H (2013) Strigolactone signaling in the endodermis is sufficient to restore root responses and involves SHORT HYPOCOTYL 2 (SHY2) activity. New Phytol 198:866-874

Kretzschmar T, Kohlen W, Sasse J, Borghi L, Schlegel M, Bachelier JB, Reinhardt D, Bours R, Bouwmeester HJ, Martinoia E (2012) A petunia ABC protein controls strigolactone-dependent symbiotic signalling and branching. Nature 483:341-344

Kume T, Sekiya N, Yano K (2006) Heterogeneity in spatial P-distribution and foraging capability by Zea mays: effects of patch size and barriers to restrict root proliferation within a patch. Ann Bot 98:1271-1277

Laplaze L, Benkova E, Casimiro I, Maes L, Vanneste S, Swarup R, Weijers D, Calvo V, Parizot B, Herrera-Rodriguez MB, Offringa R, Graham N, Doumas P, Friml J, Bogusz D, Beeckman T, Bennett M (2007) Cytokinins act directly on lateral root founder cells to inhibit root initiation. Plant Cell 19:3889-3900

Lazar G, Goodman HM (2006) MAX1, a regulator of the flavonoid pathway, controls vegetative axillary bud outgrowth in Arabidopsis. Proc Natl Acad Sci USA 103:472-476

Lin H, Wang R, Qian Q, Yan M, Meng X, Fu Z, Yan C, Jiang B, Su Z, Li J, Wang Y (2009) DWARF27, an iron-containing protein required for the biosynthesis of strigolactones, regulates rice tiller bud outgrowth. Plant Cell 21:1512-1525

Liu W, Wu C, Fu Y, Hu G, Si H, Zhu L, Luan W, He Z, Sun Z (2009) Identification and characterization of HTD2: a novel gene negatively regulating tiller bud outgrowth in rice. Planta 230:649-658

Liu W, Kohlen W, Lillo A, Op den Camp R, Ivanov S, Hartog M, Limpens E, Jamil M, Smaczniak C, Kaufmann K, Yang W-C, Hooiveld GJEJ, Charnikhova T, Bouwmeester HJ, Bisseling T, Geurts R (2011) Strigolactone biosynthesis in Medicago truncatula and rice requires the symbiotic GRAS-type transcription factors NSP1 and NSP2. Plant Cell 23:3853-3865

López-Bucio J, Cruz-Ramírez A, Herrera-Estrella L (2003) The role of nutrient availability in regulating root architecture. Curr Opin Plant Biol 6:280-287

Lorbiecke R, Sauter M (1999) Adventitious root growth and cellcycle induction in deepwater rice. Plant Physiol 119:21-29

Maillet F, Poinsot V, André O, Puech-Pagès V, Haouy A, Gueunier M, Cromer L, Giraudet D, Formey D, Niebel A, Martinez EA, Driguez H, Bécard G, Dénarié J (2011) Fungal lipochitooligosaccharide symbiotic signals in arbuscular mycorrhiza. Nature 469:58-63

Malamy JE, Benfey PN (1997) Organization and cell differentiation in lateral roots of Arabidopsis thaliana. Development 124:33-44

Mathesius U, Schlaman HRM, Spaink HP, Of Sautter C, Rolfe BG, Djordjevic MA (1998) Auxin transport inhibition precedes root nodule formation in white clover roots and is regulated by flavonoids and derivatives of chitin oligosaccharides. Plant $\mathrm{J}$ 14:23-34

Mayzlish-Gati E, De-Cuyper C, Goormachtig S, Beeckman T, Vuylsteke M, Brewer PB, Beveridge CA, Yermiyahu U, Kaplan Y, Enzer Y, Wininger S, Resnick N, Cohen M, Kapulnik Y, Koltai $H$ (2012) Strigolactones are involved in root response to low phosphate conditions in Arabidopsis. Plant Physiol 160:1329-1341

Morris SE, Turnbull CGN, Murfet IC, Beveridge CA (2001) Mutational analysis of branching in pea. Evidence that rmsl and $r m s 5$ regulate the same novel signal. Plant Physiol 126:1205-1213

Moscatiello R, Squartini A, Mariani P, Navazio L (2010) Flavonoidinduced calcium signalling in Rhizobium leguminosarum bv. viciae. New Phytol 188:814-823

Naija S, Elloumi N, Ammar S, Kevers C, Dommes J (2009) Involvement of polyamines in the adventitious rooting of micropropagated shoots of the apple rootstock MM106. In Vitro Cell Dev Biol Plant 45:83-91 
Napoli C (1996) Highly branched phenotype of the petunia dad1-1 mutant is reversed by grafting. Plant Physiol 111:27-37

Nelson DC, Scaffidi A, Dun EA, Waters MT, Flematti GR, Dixon KW, Beveridge CA, Ghisalberti EL, Smith SM (2011) F-box protein MAX2 has dual roles in karrikin and strigolactone signaling in Arabidopsis thaliana. Proc Natl Acad Sci USA 108:8897-8902

Oldroyd GED, Murray JD, Poole PS, Downie JA (2011) The rules of engagement in the legume-rhizobial symbiosis. Annu Rev Genet 45:119-144

Pei W, Du F, Zhang Y, He T, Ren H (2012) Control of the actin cytoskeleton in root hair development. Plant Sci 187:10-18

Pellerin S, Mollier A, Plénet D (2000) Phosphorus deficiency affects the rate of emergence and number of maize adventitious nodal roots. Agron J 92:690-697

Pérez-Torres C-A, López-Bucio J, Cruz-Ramírez A, Ibarra-Laclette E, Dharmasiri S, Estelle M, Herrera-Estrella L (2008) Phosphate availability alters lateral root development in Arabidopsis by modulating auxin sensitivity via a mechanism involving the TIR1 auxin receptor. Plant Cell 20:3258-3272

Pitts RJ, Cernac A, Estelle M (1998) Auxin and ethylene promote root hair elongation in Arabidopsis. Plant J 16:553-560

Porcel R, Aroca R, Ruiz-Lozano JM (2012) Salinity stress alleviation using arbuscular mycorrhizal fungi. A review. Agron Sustain Dev 32:181-200

Prayitno J, Rolfe BG, Mathesius U (2006) The ethylene-insensitive sickle mutant of Medicago truncatula shows altered auxin transport regulation during nodulation. Plant Physiol 142:168-180

Proust H, Hoffmann B, Xie X, Yoneyama K, Schaefer DG, Yoneyama K, Nogué F, Rameau C (2011) Strigolactones regulate protonema branching and act as a quorum sensing-like signal in the moss Physcomitrella patens. Development 138:1531-1539

Rasmussen A, Hunt MA (2010) Ageing delays the cellular stages of adventitious root formation in pine. Aust For 73:41-46

Rasmussen A, Mason MG, De Cuyper C, Brewer PB, Herold S, Agusti J, Geelen D, Greb T, Goormachtig S, Beeckman T, Beveridge CA (2012) Strigolactones suppress adventitious rooting in Arabidopsis and pea. Plant Physiol 158:1976-1987

Rightmyer AP, Long SR (2011) Pseudonodule formation by wild-type and symbiotic mutant Medicago truncatula in response to auxin transport inhibitors. Mol Plant Microbe Interact 24:1372-1384

Ruyter-Spira C, Bouwmeester H (2012) Strigolactones affect development in primitive plants. The missing link between plants and arbuscular mycorrhizal fungi? New Phytol 195:730-733

Ruyter-Spira C, Kohlen W, Charnikhova T, van Zeijl A, van Bezouwen L, de Ruijter N, Cardoso C, Lopez-Raez JA, Matusova R, Bours R, Verstappen F, Bouwmeester H (2011) Physiological effects of the synthetic strigolactone analog GR24 on root system architecture in Arabidopsis: another belowground role for strigolactones? Plant Physiol 155:721-734

Shinohara N, Taylor C, Leyser O (2013) Strigolactone can promote or inhibit shoot branching by triggering rapid depletion of the auxin efflux protein PIN1 from the plasma membrane. PLoS Biol 11:e1001474

Snowden KC, Simkin AJ, Janssen BJ, Templeton KR, Loucas HM, Simons JL, Karunairetnam S, Gleave AP, Clark DG, Klee HJ (2005) The Decreased apical dominance1/Petunia hybrida CAROTENOID CLEAVAGE DIOXYGENASE8 gene affects branch production and plays a role in leaf senescence, root growth, and flower development. Plant Cell 17:746-759

Soto MJ, Fernández-Aparicio M, Castellanos-Morales V, GarcíaGarrido JM, Ocampo JA, Delgado MJ, Vierheilig H (2010)
First indications for the involvement of strigolactones on nodule formation in alfalfa (Medicago sativa). Soil Biol Biochem 42:383-385

Stepanova AN, Yun J, Likhacheva AV, Alonso JM (2007) Multilevel interactions between ethylene and auxin in Arabidopsis roots. Plant Cell 19:2169-2185

Stirnberg P, Furner IJ, Leyser HMO (2007) MAX2 participates in an SCF complex which acts locally at the node to suppress shoot branching. Plant J 50:80-94

Swarup R, Perry P, Hagenbeek D, Van Der Straeten D, Beemster GTS, Sandberg G, Bhalerao R, Ljung K, Bennett MJ (2007) Ethylene upregulates auxin biosynthesis in Arabidopsis seedlings to enhance inhibition of root cell elongation. Plant Cell 19:2186-2196

Tsuchiya Y, Vidaurre D, Toh S, Hanada A, Nambara E, Kamiya Y, Yamaguchi S, McCourt P (2010) A small-molecule screen identifies new functions for the plant hormone strigolactone. Nat Chem Biol 6:741-749

Umehara M, Hanada A, Yoshida S, Akiyama K, Arite T, TakedaKamiya N, Magome H, Kamiya Y, Shirasu K, Yoneyama K, Kyozuka J, Yamaguchi S (2008) Inhibition of shoot branching by new terpenoid plant hormones. Nature 455:195-200

van Noorden GE, Ross JJ, Reid JB, Rolfe BG, Mathesius U (2006) Defective long-distance auxin transport regulation in the Medicago truncatula super numeric nodules mutant. Plant Physiol 140:1494-1506

Vanneste S, De Rybel B, Beemster GTS, Ljung K, De Smet I, Van Isterdael G, Naudts M, Iida R, Gruissem W, Tasaka M, Inzé D, Fukaki H, Beeckman T (2005) Cell cycle progression in the pericycle is not sufficient for SOLITARY ROOT/IAA14-mediated lateral root initiation in Arabidopsis thaliana. Plant Cell 17:3035-3050

Waters MT, Smith SM, Nelson DC (2011) Smoke signals and seed dormancy: where next for MAX2? Plant Signal Behav 6:1418-1422

Waters MT, Nelson DC, Scaffidi A, Flematti GR, Sun YK, Dixon KW, Smith SM (2012) Specialisation within the DWARF14 protein family confers distinct responses to karrikins and strigolactones in Arabidopsis. Development 139:1285-1295

Williamson LC, Ribrioux SPCP, Fitter AH, Leyser HMO (2001) Phosphate availability regulates root system architecture in Arabidopsis. Plant Physiol 126:875-882

Wu C, Dickstein R, Cary AJ, Norris JH (1996) The auxin transport inhibitor $N$-(1-naphthyl)phthalamic acid elicits pseudonodules on nonnodulating mutants of white sweetclover. Plant Physiol 110:501-510

Xie X, Yoneyama K, Yoneyama K (2010) The strigolactone story. Annu Rev Phytopathol 48:93-117

Yoneyama K, Yoneyama K, Takeuchi Y, Sekimoto H (2007) Phosphorus deficiency in red clover promotes exudation of orobanchol, the signal for mycorrhizal symbionts and germination stimulant for root parasites. Planta 225:1031-1038

Yoneyama K, Xie X, Kim HI, Kisugi T, Nomura T, Sekimoto H, Yokota T, Yoneyama K (2012) How do nitrogen and phosphorus deficiencies affect strigolactone production and exudation? Planta 235:1197-1207

Zhang S, Li G, Fang J, Chen W, Jiang H, Zou J, Liu X, Zhao X, Li X, Chu C, Xie Q, Jiang X, Zhu L (2010) The interactions among DWARF10, auxin and cytokinin underlie lateral bud outgrowth in rice. J Integr Plant Biol 52:626-638 